(c) Elsevier/INRA

Original article

\title{
Long telomeres in the polytene chromosomes of Drosophila melanogaster are associated with amplification of subtelomeric repeat sequences
}

\author{
ON Danilevskaya ${ }^{1 *}$, GE Lapta ${ }^{2}$ \\ ${ }^{1}$ USSR Academy of Sciences, Institute of Molecular Genetics, 123182 Moscow; \\ 2 Kharkov State University, Kharkov, USSR
}

(Received 23 April 1990; accepted 7 November 1990)

\begin{abstract}
Summary - The T-32 strain of Drosophila melanogaster, which was originally selected for heat resistance, is characterized by having exceptionally long tips in chromosomes $X$ and $2 L$ in the polytene salivary gland chromosomes. Six sublines differing in telomere length in different chromosomes were selected at an elevated temperature $\left(32^{\circ} \mathrm{C}\right)$. The morphological characteristics of the telomeres in each subline are stable for a number of generations at $23^{\circ} \mathrm{C}$. The additional chromatin in the long telomeres effectively hybridizes with a DNA sequence designated Dm665, which is related to the He-T family of heterochromatin- and telomere-associated sequences. The short telomeres show weak or no hybridization with the Dm665 fragment. This result implies that at least part of the morphological changes in telomere length in polytene chromosomes results from different degrees of amplification of the telomere-associated sequences.
\end{abstract}

$D$ melanogaster / polytene chromosome / telomere length / repeat sequence / amplification

Résumé - Les télomères longs des chromosomes polytènes de Drosophila melanogaster sont associés à l'amplification de séquences répétées subtélomériques. La souche T-32 de $\mathrm{D}$ melanogaster, sélectionnée à l'origine pour sa résistance à la chaleur, se caractérise par des extrémités exceptionnellement allongées de ses chromosomes $X$ et $2 L$ (chromosomes polytènes des glandes salivaires). Six sous-lignées, différant par la longueur des télomères de différents chromosomes, ont été sélectionnées à température élevée $\left(32^{\circ} \mathrm{C}\right)$. Dans chacune des sous-lignées, les caractéristiques des télomères demeurent stables pendant plusieurs générations $\grave{a} 23^{\circ} \mathrm{C}$. La chromatine additionnelle des longs télomères s'hybride avec une séquence d'ADN (Dm 665) apparentée à la famille He$T$ de séquences associées à l'hétérochromatine et aux télomères. Les télomères courts présentent une hybridation faible ou nulle avec le fragment Dm 665. Ces résultats indiquent qu'au moins une partie des modifications morphologiques de la longueur des télomères des chromosomes polytènes peut être altribuée à des variations du degré d'amplification des séquences associées aux télomères.

$D$ melanogaster / chromosome polytène / longueur de télomère / séquence répétée / amplification

* Correspondence and reprints 


\section{INTRODUCTION}

Polytene chromosomes from different wildtype strains of Drosophila melanogaster can differ by the amount of chromatin in the telomeres of different chromosomes. Morphologically, some telomeres appear long and others short (Roberts, 1979). The additional chromatin in the long telomeres has no well defined band structure but can vary in size and general morphology. The morphological peculiarities of telomeres can persist for a number of generations and serve as cytological markers in certain wildtype stocks (Lapta and Shakhbazov, 1986).

It seemed possible that the long and short telomeres might result from differential amplification of one or more subtelomeric repeat sequences found in the Drosophila genome. This hypothesis was examined using the cloned DNA fragment Dm665 and the 10.5 -kb insert of the phage $\lambda 17$, which contains Dm665 and 2 copies of the transposable element hoppel (Danilevskaya et al, 1984). The Dm665 fragment is an AT-rich repeated sequence found at the tips of chromosomes and in heterochromatin, particularly in the $Y$ chromosome. It is related to the He- $\mathrm{T}$ family of heterochromatic-telomeric sequences (Young et al, 1983; Traverse and Pardue, 1989). The Drosophila strains used in the analysis were derived from a stock designated $\mathrm{T}-32$, which is characterized by the presence of additional chromatin in the telomeres of the chromosome arms $X$ and $2 L$. T-32 originates from flies collected in N'Djamena (Chad, Central Africa) that were subjected to artificial selection for increased heat tolerance (Tikhomirova and Belyatskaya, 1980). Flies of the $\mathrm{T}-32$ strain are exceptional in that they can develop from egg to imago at $32^{\circ} \mathrm{C}$ without losing their fertility. Furthermore, when T-32 is reared at high temperatures, sublines can be obtained that have additional chromatin at the tips of one or more chromosome arms in the salivary gland chromosomes. The mechanism of origin of these telomeric variants is uncertain, but the telomere morphology is stable in successive generations when the sublines are reared at $23^{\circ} \mathrm{C}$. In this way we obtained 6 sublines of T-32 that exhibited different telomere morphologies, including sublines that differ from one another by long and short telomere variants of the same chromosome arm.

We have found that the additional chromatin of the long telomeres intensively hybridizes with labeled Dm665 sequences. The short telomeres show a much weaker hybridization (or none at all) with the probe sequences. We conclude that the morphological changes in the telomeres of polytene chromosomes are caused by different degrees of amplification of at least Dm665 and perhaps other telomereassociated sequences.

\section{MATERIALS AND METHODS}

\section{Drosophila stocks}

The heat-resistant strain T-32, kindly provided by MM Tikhomirova, served as starting material. One generation of flies was reared at $32^{\circ} \mathrm{C}$, and a number of sublines were created by mating 2 males and 2 females chosen at random. The sublines were maintained at $23^{\circ} \mathrm{C}$. The telomere morphology of the sublines 
was examined in polytene chromosome squashes prepared by standard procedure (Atherton and Gall, 1972) with minor modifications.

\section{In situ hybridization}

Polytene chromosomes of salivary glands of Drosophila larvae were prepared according to Gall and Pardue (1971). The DNA probes were labeled with tritiated thymidine by nick translation. The $2.4-\mathrm{kb}$ Dm665 fragment was obtained by subcloning the Dm665 HindIII fragment from plasmid p665 into pBR322 (Danilevskaya et al, 1984). A larger fragment homologous to Dm665 was obtained from a $10.5-\mathrm{kb}$ insert in bacteriophage $\lambda 17$. This fragment includes not only sequences homologous to Dm665 but also 2 copies of a novel transposable element designated hoppel (Kurenova, personal communication). Hybridization sites of the probes were determined in 5-10 individuals in each subline. Although the figures in this paper were prepared from material hybridized with $\lambda 17$ for the sake of signal intensity, the same results were obtained when the material was hybridized with Dm665. Hence we attribute the pattern of hybridization signals obtained with $\lambda 17$ as coming at least from the Dm665 sequences it contains and perhaps also from the hoppel elements.

\section{RESULTS}

\section{Selection of T-32 sublines with different telomere morphology}

The polytene chromosomes of strain T-32 have additional blocks of chromatin in the telomeres of the chromosome arms $X$ and $2 L$. When $T-32$ is reared at high temperatures $\left(32^{\circ} \mathrm{C}\right)$, sublines can be isolated that differ in telomere morphology, and these variants are stably maintained at $23^{\circ} \mathrm{C}$. The generation of these stable sublines allowed us to study sublines with additional telomeric chromatin in different chromosomes, and we were able to obtain different combinations of chromosomes with long and short telomeres in the same genetic background. The morphology of the long and short telomeres found at the tips of the $X, 2 L$, and $3 R$ is illustrated in figure 1. Six sublines differing in telomere morphology with particular combinations of long, intermediate, and short telomeres are diagrammed in figure 2 (lines A-F). The telomeres in some lines are not as long as the long telomeres, and not as short as the short telomeres, and we call them "intermediate" only to indicate this ambiguity. Flies with short telomeres in all the chromosomes (exemplified by subline E) occurred with the lowest frequency. Such sublines are somewhat unstable and quickly acquire long telomeres in some chromosomes, most often in the right arm of chromosome 3 (eg, subline F).

\section{In situ hybridization of larval salivary gland polytene chromosomes from different $T$-32 sublines with telomere-associated probes}

Polytene chromosomes from the 6 sublines with morphologically different telomeres were subjected to in situ hybridization with 2 probes containing sequences homologous to the subtelomeric repeat designated Dm665 (Danilevskaya et al, 1984). Parallel hybridizations with Dm665 and $\lambda 17$ probes were performed on salivary 

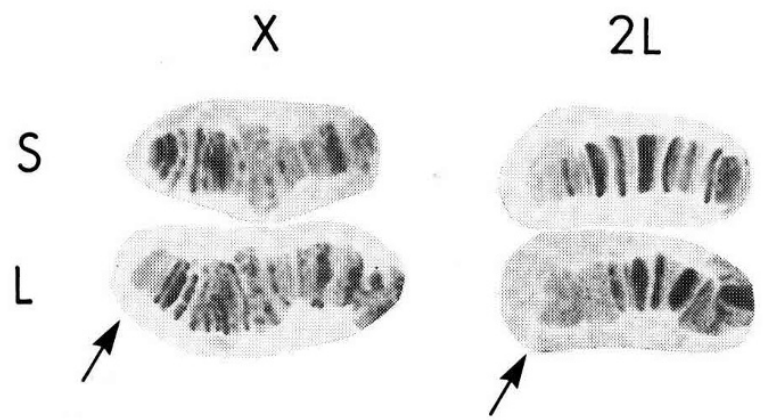

\section{$3 R$}

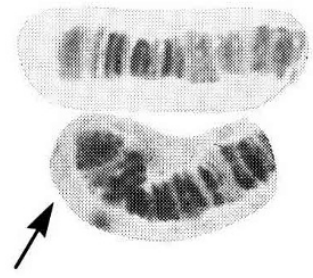

Fig 1. Morphology of long (L) and short (S) telomeres. Arrows indicate additional chromatin.



Fig 2. Schematic representation of telomere morphology in sublines A-F derived from $\mathrm{T}-32$. The length and labeling intensity of the telomeres is indicated by the length of the shaded bars. The open bars (not to scale) represent the rest of the chromosome.

glands from the same larva. More intense hybridizations of the same telomeres were obtained with a $10.5-\mathrm{kb}$ sequence cloned in bacteriophage $\lambda 17$, but the presence of the mobile element hoppel in the $\lambda$ phage also results in hybridization with the chromocenter and several sites internal to the chromosomes arms.

A diagrammatic summary of the labelling data with the telomere-associated probes is presented in figum 2. This composite is based on results like those illustrated in figures 3-6, which show larval salivary gland polytene chromosomes from sublines A, B, C and D, respectively, with and without hybridization. Figures 3 (subline A) and 5 (subline C) show the telomeric regions only, while figures 4 (subline B) and 6 (subline D) show the entire euchromatic complement. The arrows indicate the additional chromatin found in the long telomeres.

In all the sublines the long $X$ and $2 L$ telomeres carrying additional chromatin can be seen to hybridize effectively with the telomere-associated probes. The appearance of additional chromatin in the $3 \mathrm{R}$ telomere (sublines $\mathrm{D}$ and $\mathrm{F}$ ) leads to enhanced hybridization of this chromosome tip (figs 1,6). Note that these sublines also exhibit some hybridization with the $3 L$ telomere, which is unlabeled in the other sublines. 


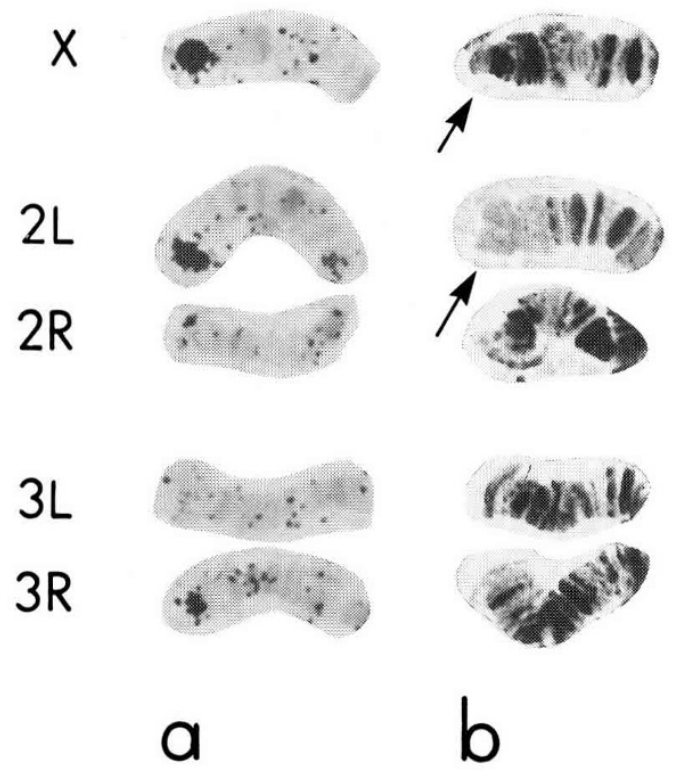

Fig 3. Telomeric regions of larval salivary gland polytene chromosomes from subline A. (a) After hybridization with telomere-associated probe $\lambda 17$. (b) Intact chromosomes stained with acetic orcein. The arrows indicate additional chromatin. The chromosomes have been deliberately overstained in order to emphasize the tips. Their identity was determined by examination of the banding patterns in regions proximal to those shown in the photographs.

All the short-telomere variants of the $X$ chromosome also hybridize with $\lambda 17$, but less intensely than $X$ chromosomes with the long telomeres. The short $2 L$ telomeres in sublines $\mathrm{B}, \mathrm{E}$ and $\mathrm{F}$ do not hybridize to a detectable level with $\lambda 17$ (fig 4), nor do the short $3 L$ telomeres in sublines A, B, C and $\mathrm{E}$ (figs 4,5 ). The pattern of hybridization is especially clear in heterozygotes produced by crossing the sublines, in which only one of the homologues has additional chromatin in the $2 L$ telemore. Figure 7 shows the intensive labelling of the long $2 L$ homologue as compared with the hardly noticeable label in the homologue with the short telomere. This result demonstrates that the short telomeres do contain a small number of copies of telomere-associated sequences homologous to $\lambda 17$. These are revealed by an appropriately labeled probe, even though they are not associated with morphologically visible additional chromatin. Overall, our results imply that the labeling intensity of telomeres when hybridized with the telomere-associated probe Dm665 or $\lambda 17$ directly correlates with the presence of additional chromatin.

\section{DISCUSSION}

Morphological differences among the telomeric regions of polytene chromosomes in different Drosophila strains have been known for some time (Roberts, 1979). The long and short telomeres differ by additional chromatin that may occur in different 

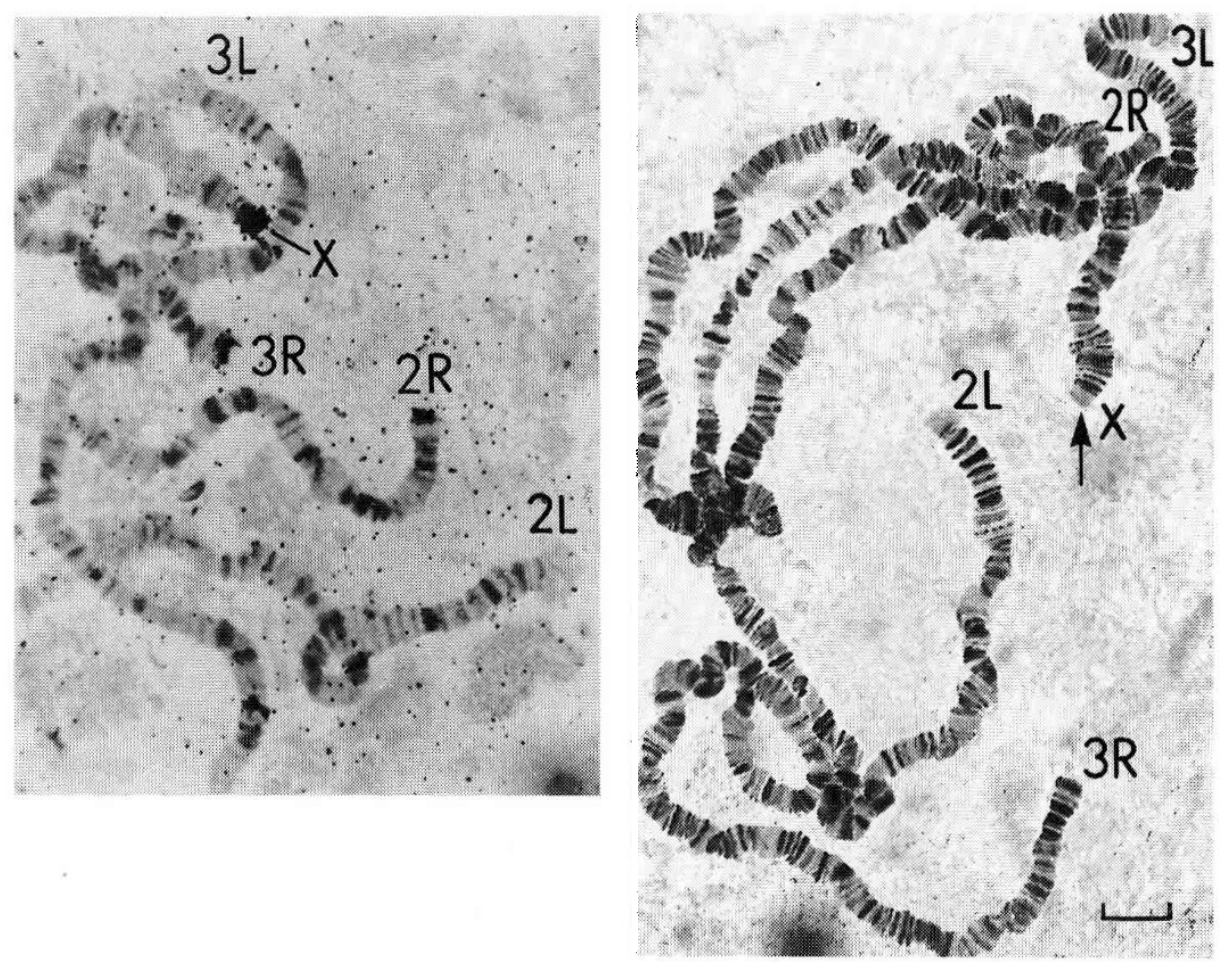

Fig 4. Larval salivary gland polytene chromosomes from subline B. (a) After hybridization with telomere-associated probe $\lambda 17$. (b) Stained with acetic orcein. Bar represents $10 \mu \mathrm{m}$.

chromosomes. However, most of the differences have been observed in strains of very diverse genetic backgrounds. In our study the sublines differing in telomere length all derive from T-32 and so have a common genetic background.

The obvious next step in the analysis of morphological variation in telomeres is to determine what DNA sequences comprise the additional chromatin. The molecular structure of telomeres is under intensive investigation in a number of systems, and a general pattern seems to have emerged (Blackburn and Szostak, 1984). The ends of chromosomal DitA consist of simple, tandemly satellite-like sequences that are species specific. Adjoining these are telomere-associated repeats of varying length, often organized in tandem arrays. The sequences at the chromosome tips in Drosophila have not been established, but telomere-associated fragments have been cloned in several laboratories (Rubin, 1978; Young et al, 1983; Renkawitz-Pohl and Bialojan, 1984). They belong to the He-T family of sequences found in the telomeric and heterochromatic regions of the genome (Young et al, 1983; Traverse and Pardue, 1989; Biessmann et al, 1990; Levis, personal communication). The fragment Dm665 (Danilevskaya et al, 1984), which is a member of the He-T family of repetitive sequences, is located in the $Y$ chromosome and shares homology with sequences located in subtelomeric regions (Danilevskaya et al, 1984; and unpub- 

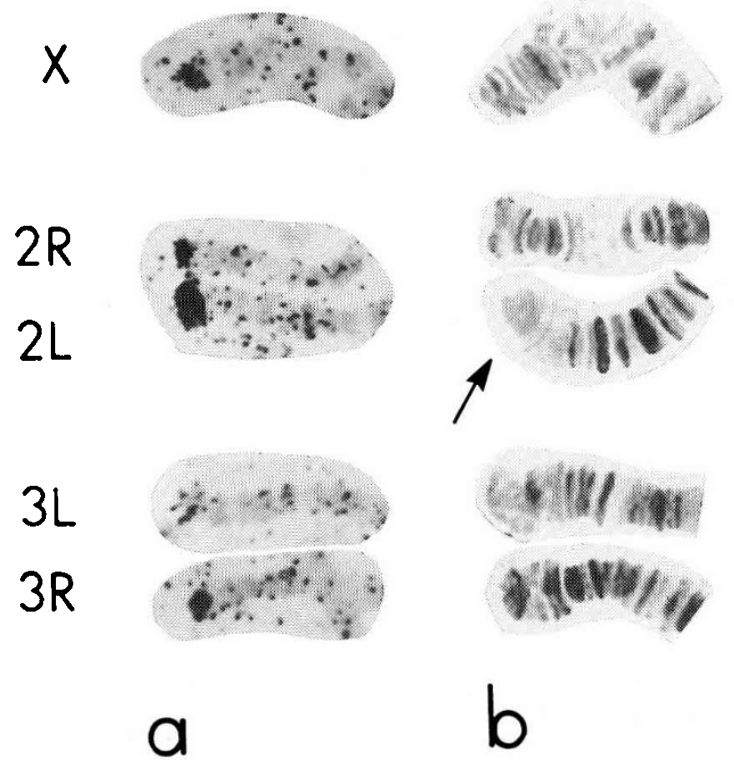

b

Fig 5. Telomeric regions of larval salivary gland polytene chromosomes from subline C. (a) After hybridization with telomere-associated probe $\lambda 17$. (b) Stained with acetic orcein.
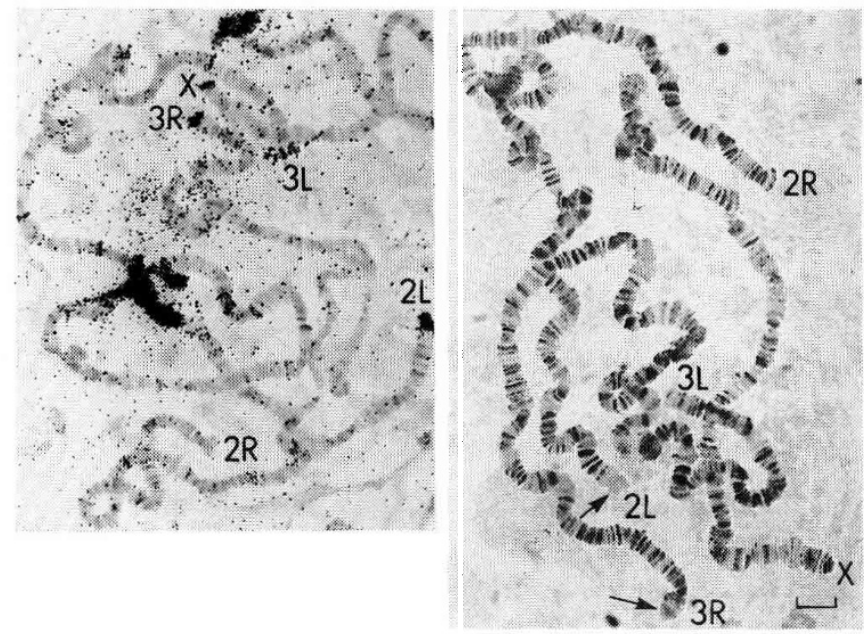

Fig 6. Larval salivary gland polytene chromosomes from subline D. (a) After hybridization with telomere-associated probe $\lambda 17$. (b) Stained with acetic orcein. Bar represents $10 \mu \mathrm{m}$. 


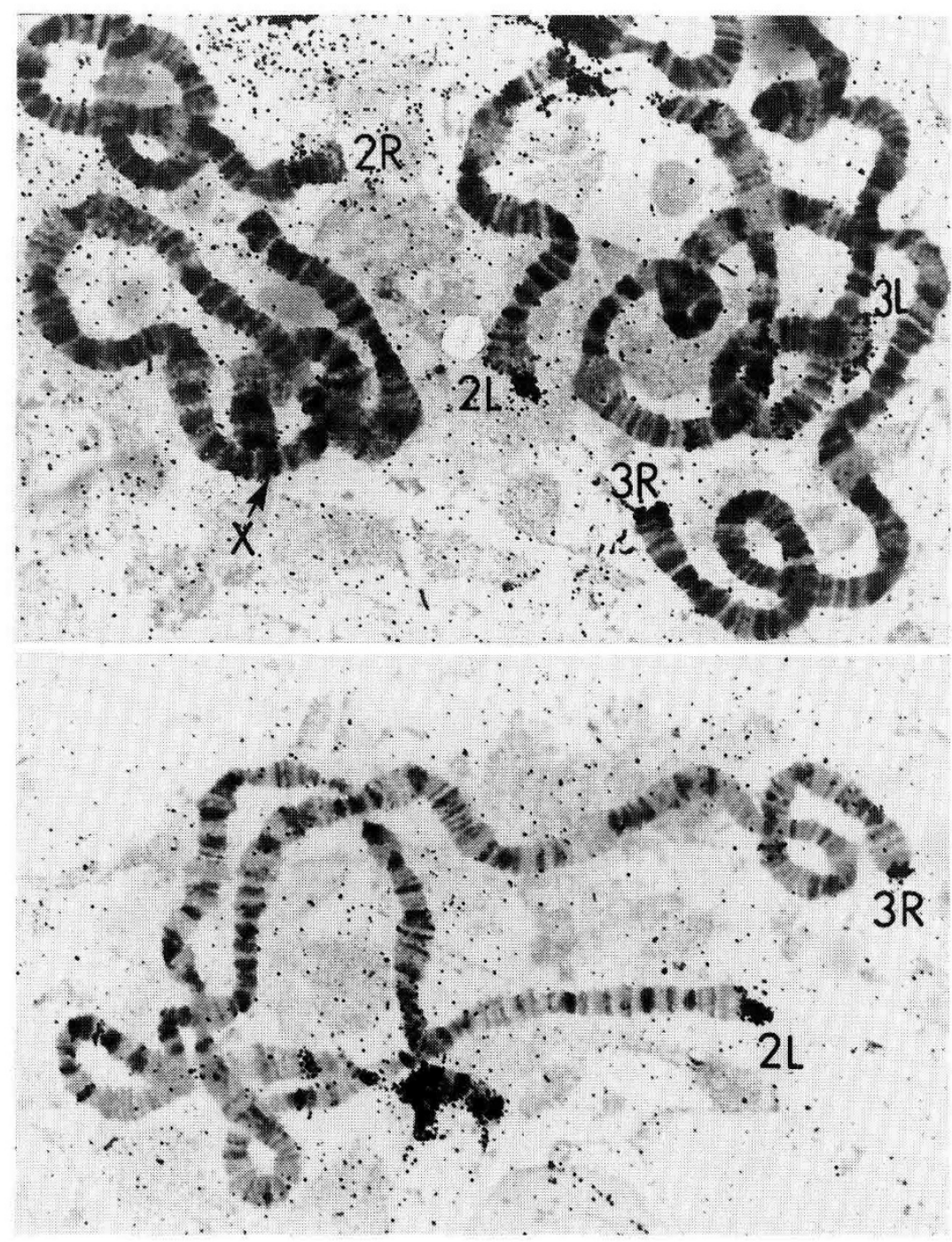

Fig 7. Larval salivary gland chromosomes from 2 individuals, produced by crossing, that are heterozygous for long and short variants of the telomere of $2 L$. The hybridization was performed with the telomere-associated probe in phage $\lambda 17$. Note that the $2 L$ chromosome is heterozygous for the long and short telomere variant.

lished observations). The telomere-associated sequences also frequently contain mobile elements of various kinds (Danilevskaya, unpublished observations). One of these mobile elements, designated hoppel, is frequently associated with the He-T family and is present in the $\lambda 17$ clone (Kurenova, personal communication). 
The Drosophila melanogaster strain T-32, originally selected for increased heat tolerance, has provided an excellent experimental model for obtaining sublines with altered telomere morphology. Rearing T-32 at $32^{\circ} \mathrm{C}$ results in a high frequency of alterations in telomere morphology, and sublines differing in telomere morphology can be stably maintained at $23^{\circ} \mathrm{C}$.

Hybridization of labeled telomere-associated probes with salivary gland polytene chromosomes from different sublines of T-32 demonstrated that sequences homologous to Dm665 are amplified in the long telomeres. The short telomeres show a far less intense hybridization, and in some cases no detectable hybridization. Hence, the morphologically visible additional chromatin in the long telomeres results from the amplification of at least Dm665 and perhaps other telomere-associated DNA sequences.

Roberts (1979) proposed that the long and short telomeres result from a heritable difference in the location of a transition zone between polytene and nonpolytene chromatin. Our results suggest that the long telomeres may result from the amplification of telomere-associated sequences. This may occur in the germline and merely be observed in the polytene chromosomes, or the amplification may occur specifically in the salivary gland and perhaps other polytene chromosomes. A critical test will require direct in situ comparison of salivary gland chromosomes and metaphase chromosomes from other tissues, in order to allow an estimation of the number of copies of Dm665 that occur in the tips of metaphase chromosomes in diploid tissues.

The effect of temperature on the morphology and pattern of hybridization of telomeres in sublines selected from T-32 is highly unusual. The result suggests that elevated temperature may stimulate deletion, amplification, or rearrangement of the telomere-associated gene family. Except for the effect of elevated temperature, the processes involved may be entirely conventional, for example, unequal crossing over or gene conversion. However, the He-T gene family may be subject to unprecedented processes. For example, Traverse and Pardue (1988) and Biessmann et al (1990) have described the de novo addition of He-T family sequences to the tips of broken chromosomes. These additions serve the role of telomeres in stabilizing the tips against the loss of DNA during replication. Moreover, the addition of the He-T sequences to the ends of broken chromosomes does not require sequence homology to the ends. What processes are involved have not yet been identified, but it is possible that the process is promoted by elevated temperatures in the T-32 strain.

\section{ACKNOWLEDGMENTS}

The authors thank VG Nikiforov and VG Shakhbazov for support and encouragement, MM Tikhomirova for strain T-32 stock, ES Belyaeva and BA Leibovich for their aid and advice, and DL Hartl for his help and encouragement.

\section{REFERENCES}

Atherton D, Gall JG (1972) Salivary gland squashes for in situ nucleic acid hybridization studies. Dros Inf Serv 49, 131-133 
Blackburn EH, Szostak JW (1984) The molecular structure of centromeres and telomeres. Ann Rev Biochem 53, 163-210

Biessmann H, Mason JM, Ferry K, d'Hulst M, Valgeirsdottir K, Traverse KL, Pardue ML (1990) Addition of telomere-associated He-T DNA sequences "heals" broken chromosome ends in Drosophila. Cell 61, 663-673

Danilevskaya ON, Kurenova EV, Leibovich BA, Shevelev AYu, Bass IA, Khesin RB (1984) Telomeres and P-element of Drosophila melanogaster contain sequences that replicate autonomously in Saccharomyces cerevisiae. Mol Gen Genet 197, 342-344 Gall JG, Pardue ML (1971) Nucleic acid hybridization in cytological preparations. Methods Enzymol 21, 470-480

Lapta GE, Shakhbazov VG (1986) Analysis of specificity of ectopic conjugation of telomeric ends of Drosophila melanogaster polytene chromosomes. Genetika (USSR) 22, 787-792

Renkawitz-Pohl R, Bialojan S (1984) A DNA sequence of Drosophila melanogaster with a different telomeric distribution. Chromosoma 89, 206-211

Roberts PA (1979) Rapid change of chromomeric and pairing patterns of polytene chromosome tips in Drosophila melanogaster: migration of polytene-nonpolytene transition zone? Genetics 92, 861-882

Rubin GM (1978) Isolation of a telomeric DNA sequence from Drosophila melanogaster. Cold Spring Harbor Symp Quant Biol 42, 1041-1046

Tikhomirova MM, Belyatskaya OYa (1980) Modifying effect of extremal temperature depending on the organism adaptation to this factor on the action of radiation. I. Characterization of a Drosophila stock adapted to high temperature. Genetika (USSR) 16, 115-122

Traverse KL, Pardue ML (1988) A spontaneously opened ring chromosme of Drosophila melanogaster has acquired He-T DNA sequences at both new telomeres. Proc Natl Acad Sci USA 85, 8116-8120

Traverse KL, Pardue ML (1989) Studies of He-T DNA sequences in the pericentric regions of Drosophila chromosomes. Chromosoma 97, 261-271

Young BS, Pession A, Traverse KL, Frech C, Pardue ML (1983) Telomere regions in Drosophila share complex DNA sequences with pericentric heterochromatin. Cell $34,85-94$ 